\title{
INTEGRAL REPRESENTATIONS FOR MARKOV TRANSITION PROBABILITIES
}

\author{
BY DAVID G. KENDALL
}

Communicated by Paul R. Halmos, July 14, 1958

The following results (and one or two others like them) are proved in [3]; the continuous-parameter analogues will appear in [4] and some applications will be discussed in [5]. The proofs are based on Sz.-Nagy's group of theorems [9] which show how a discrete- or continuous-parameter semigroup of contraction operators on Hilbert

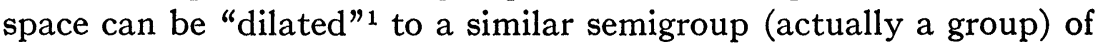
unitary operators acting on a larger Hilbert space. The WintnerStone theorems on the spectral representation of discrete/continuousparameter groups of unitary operators then lead easily to integral representations for the Markov transition probabilities which appear in the matrix elements of the operator to be dilated.

I. An irreducible Markov chain with a denumerable set of states possesses at least one "positive sub-invariant measure" $\left\{m_{j}: j=1,2, \cdots\right\}$; i.e., there exist positive finite real numbers $m_{j}$ such that

$$
\sum_{\alpha} m_{\alpha} p_{\alpha k} \leqq m_{k} \quad(k=1,2, \cdots),
$$

where $p_{j k}$ is the probability that a single transition from state $\mathcal{E}_{j}$ will lead directly to the state $\varepsilon_{k}$. The numbers $m_{j}$ need not be unique, even if we require the normalization $m_{1}=1$.

II. If $\left\{m_{j}: j=1,2, \cdots\right\}$ is a given positive sub-invariant measure associated with an irreducible Markov chain then the $n$-step transition probabilities for the latter can be uniquely represented in the form

$$
p_{j k}^{n}=\left(m_{k} / m_{j}\right)^{1 / 2} \oint e^{i n \theta}{ }^{i j k}(d \theta) \quad(n=0,1,2, \cdots)
$$

where the complex-valued Borel measures $\mu_{j k}$ are supported by the circumference $\Gamma$ of unit radius and are required to satisfy the Hermitean condition

$$
\mu_{k j}=\mu_{j k} \quad(j, k=1,2, \cdots) .
$$

The atoms of $\mu_{j k}$ (of which there are none unless the chain is positiverecurrent) are located at the $d$ dth roots of unity on $\Gamma$ (where $d$ is the

1 The terminology is due to P. R. Halmos (see [9]). 
period of the chain) and the nonatomic component of $\mu_{j k}$ is absolutely continuous with respect to Lebesgue measure. This result shows that the rate-of-convergence problem for the $p_{j k}^{n}$ (as $n$ tends to infinity) can be reduced to one involving the asymptotic behavior of the Fourier constants of a summable function.

III. Either ALL or NONE of the states of an irreducible aperiodic Markov chain have the geometric convergence property,

$$
\left|p_{j j}^{n}-L_{j}\right| \leqq A_{j} \rho_{j}^{n} \text { for all } n \text {, where } 0 \leqq \rho_{j}<1 \text {. }
$$

When the alternative "all" holds then the nondiagonal transition probabilities $p_{j k}^{n}(j \neq k)$ also converge geometrically to their ergodic limits. Such a "geometrically ergodic" chain must be either transient or positiverecurrent; it cannot be null-recurrent.

IV. Let us say that an irreducible Markov chain is reversible with respect to a positive sub-invariant measure $\left\{m_{j}: j=1,2, \cdots\right\}$ satisfying (1) above if

$$
m_{j} p_{j k}=m_{k} p_{k j} \text { for all } j \text { and } k .
$$

Such a measure if it exists is unique (if we put $m_{1}=1$ ) and it must be invariant (i.e. equality holds in (1)). A necessary and sufficient condition for an irreducible Markov chain to be reversible with respect to one of its positive sub-invariant measures is that

$$
p_{j k_{1}} p_{k_{1} k_{2}} \cdots p_{k_{r} j}=p_{j k_{r}} p_{k_{r} k_{r-1}} \cdots p_{k_{1} j}
$$

for all positive integers $r$ and all states $\varepsilon_{j}, \varepsilon_{k_{1}}, \cdots, \varepsilon_{k_{r}}$. The condition (6) (which need only be verified for $r \geqq 2$ and for all distinct states $\varepsilon_{j}, \varepsilon_{k_{1}}, \cdots, \varepsilon_{k_{r}}$ ) was given by Kolmogorov [6] as a necessary and sufficient condition for a finite Markov chain to be "reversible in time"; Theorem IV may be regarded as a generalization of his result to infinite chains. (For the special case of positive-recurrent chains this result was given by Reich [8].)

V. The bounded linear operator $T$ on the Hilbert space $l_{2}$ defined by

$$
(T x)_{k}=\sum_{\alpha} x_{\alpha}\left(m_{\alpha} / m_{k}\right)^{1 / 2} p_{\alpha k} \quad(k=1,2, \cdots)
$$

has norm less than or equal to unity, and it is self-adjoint if and only if the irreducible chain $\left\{p_{j k}: j, k=1,2, \cdots\right\}$ is reversible with respect to the positive sub-invariant measure $\left\{m_{j}: j=1,2, \cdots\right\}$ (a necessary and sufficient condition for this to be so is given by Theorem IV). The reversibility condition (6) is automatically satisfied whenever the only nonzero elements of $\left\{p_{j k}: j, k=1,2, \cdots\right\}$ lie on the principal 
or the two immediately adjacent diagonals; thus all such "random walk" type chains are reversible and determine self-adjoint operators. This fact (and the spectral theorem for bounded self-adjoint operators) now appears as the ultimate source of a series of integral representations found by Kac [1], by Ledermann and Reuter [7] and by Karlin and McGregor [2] for probability matrices associated with "random walk" and "birth and death" systems, and Theorems IV and V (and VIII below) finally delimit the area within which such "self-adjoint" integral representations are to be found. When condition (6) is not satisfied the only general procedure available is the dilation of the contraction $T$ to a unitary operator on a larger space; this is in fact how one arrives at the "unitary" integral representation of Theorem II.

VI. By means of a compactness argument it is possible to extend some of the above results to reducible chains; the following illustrates what can be achieved in this way. With each state $\varepsilon_{j}$ of a (perhaps reducible) Markov chain there is uniquely associated a probability measure $\nu_{j j}$ on the Borel subsets of the closed interval $[0, \pi]$ such that

$$
p_{j j}^{n}=\int_{0}^{\pi} \cos n \theta v_{j j}(d \theta) \quad(n \geqq 0) .
$$

The measure $\nu_{j j}$ is absolutely continuous with respect to Lebesgue measure, save for atoms at $\theta=0,2 \pi / d, 4 \pi / d, \cdots, 2[d / 2] \pi / d$ in the special case when $\mathcal{E}_{j}$ is positive-recurrent with period $d \geqq 1$. The absolutely continuous component of the measure has the density

$$
\frac{1}{\pi} \frac{1-\left|F_{j j}\left(e^{i \theta}\right)\right|^{2}}{\left|1-F_{j j}\left(e^{i \theta}\right)\right|^{2}}
$$

where $F_{j j}(z)$ is the generating function of the probability distribution of the recurrence-time for the state $\varepsilon_{j}$ (so that $\left.F_{j j}(1) \leqq 1\right)$.

VII. By means of an imbedded-chain technique one can extend much of the above to the continuous-parameter case (processes instead of chains). Thus, if we call a process irreducible when, for each fixed $j$ and $k, p_{j k}(t)$ is positive for all sufficiently large $t$, and if the usual regularity condition $p_{j k}(t) \rightarrow \delta_{j k}(t \rightarrow 0)$ is postulated, then we find that each irreducible Markov process admits a positive sub-invariant measure $\left\{m_{j}: j=1,2, \cdots\right\}$ such that

$$
\sum_{\alpha} m_{\alpha} p_{\alpha k}(t) \leqq m_{k} \quad(k=1,2, \cdots ; t \geqq 0),
$$

and if one such measure $\left\{m_{j}: j=1,2, \cdots\right\}$ is chosen then there exists 
a unique Hermitean system $\left\{\mu_{j k}: j, k=1,2, \cdots\right\}$ of complex-valued totally-finite Borel measures supported by the real line and such that

$$
p_{j k}(t)=\left(m_{k} / m_{j}\right)^{1 / 2} \int_{-\infty}^{\infty} e^{i \lambda t} \mu_{j k}(d \lambda)
$$

Each of the measures $\mu_{j k}$ is absolutely continuous with respect to Lebesgue measure save for an atom at $\lambda=0$ (which is not present unless the process is "positive-recurrent").

VIII. By arguments analogous to those referred to at IV above one can extend the theory relating "reversibility" with the self-adjoint character of the associated operators to the continuous-parameter case, and so obtain results of which the following is a sample. Let $\left\{q_{j k}: j, k=1,2, \cdots\right\}$ be a system of finite real numbers such that

$$
q_{j k} \geqq 0(j \neq k), \quad q_{j} \equiv-q_{j j} \geqq 0, \quad \sum_{\beta} q_{j \beta}=0,
$$

and such that the equations

$$
\sum_{\beta} q_{j \beta} y_{\beta}=\lambda y_{j} \quad(j=1,2, \cdots)
$$

have for some (and then for all) positive $\lambda$ no non-null bounded solution; then there will exist a unique Markov process (the Feller process generated by the $q_{j k}$ 's) such that $p_{j k}^{\prime}(0+)=q_{j k}$, and it will be irreducible if and only if every pair $j$ and $k$ of distinct positive integers can be linked by a finite chain $\left(j, h_{1}, \cdots, h_{s}, k\right)$ of positive integers such that

$$
q_{j h_{1}} q_{h_{1} h_{2}} \cdots q_{h_{\mathrm{g}} k}>0 .
$$

If the irreducibility condition is satisfied then the following condition

$$
\begin{gathered}
\mathcal{K}_{q}: \quad q_{j k_{1}} q_{k_{1} k_{2}} \cdots q_{k_{r} j}=q_{j k_{r}} q_{k_{r} k_{r-1}} \cdots q_{k_{1} j} \\
\text { for each positive integer r and for all sets } \\
\text { of positive integers }\left(j ; k_{1}, \cdots, k_{r}\right),
\end{gathered}
$$

is both necessary and sufficient for the transition probabilities of the Feller process to be (uniquely) representable in the form

$$
p_{j k}(t)=\left(m_{k} / m_{j}\right)^{1 / 2} \int_{0}^{\infty} e^{-t \tau} d G_{j k}(\tau) \quad(t \geqq 0)
$$

where $\left\{G_{j k}(\cdot): j, k=1,2, \cdots\right\}$ is a symmetric system of real-valued functions of totally-bounded variation on $[0, \infty)$ which are continuous to the left and which satisfy $G_{j k}(0)=0, G_{j k}(\infty)=\delta_{j k}$; the ratios $m_{j}: m_{k}$ 
are here uniquely determined by the equations

$$
m_{j} q_{j k}=m_{k} q_{k j} \quad(j, k=1,2, \cdots) .
$$

Not all of the Laplace-Stieltjes integral representations given by Ledermann, Reuter, Karlin and McGregor relate to irreducible Feller processes, but those which do are all instances of the above general theorem and of the remark that condition $\mathfrak{K}_{q}$ is automatically satisfied whenever the $q_{j k}$ 's vanish everywhere save on the principal and the two immediately adjacent diagonals. (For "positive-recurrent" processes the connection between $\mathscr{K}_{q}$ and reversibility was given by Reich [8].)

\section{REFERENCES}

1. M. Kac, Random walk and the theory of the Brownian motion, Amer. Math. Monthly vol. 54 (1947) pp. 369-391.

2. S. Karlin and J. L. McGregor, Representation of a class of stochastic processes, Proc. Nat. Acad. Sci. U.S.A. vol. 41 (1955) pp. 387-391.

3. D. G. Kendall, Unitary dilations of Markov transition operators, and the corresponding integral representations for transition-probability matrices, to appear in the volume Surveys in probability and statistics dedicated to Harald Cramér (edited by U. Grenander), Stockholm, Almqvist and Wiksell.

4. - Unitary dilations of one-parameter semigroups of Markov transition operators, and the corresponding integral representations for Markov processes with a countable infinity of states, to appear.

5. - Geometric ergodicity in the theory of queues, to appear.

6. A. N. Kolmogorov, Zur Theorie der Markoffschen Ketten, Math. Ann. vol. 112 (1936) pp. 155-160.

7. W. Ledermann and G. E. H. Reuter, Spectral theory for the differential equations of simple birth and death processes, Philos. Trans. Roy. Soc. London Ser. A vol. 246 (1954) pp. 321-369.

8. E. Reich, Waiting times when queues are in tandem, Ann. Math. Statist. vol. 28 (1957) pp. 768-773.

9. B. Sz.-Nagy, Prolongements des transformations de l'espace de Hilbert qui sortent de cet espace, Appendix, 1955, to F. Riesz and B. Sz.-Nagy, Leçons d'analyse fonctionnelle, Budapest, 1952.

Magdalen College, Oxford 